\title{
An Unusual Presentation of Small Bowel Intussusception
}

\author{
Yinglin Gao, DO; Cheikh Talal El Imad, MD²; Hai Song Kim, DO3; Vivek Gumaste, MD $^{2}$ \\ ${ }^{1}$ Loma Linda University Medical Center, Loma Linda, CA \\ ${ }^{2}$ Staten Island University Hospital, Staten Island, NY \\ ${ }^{3}$ The University of Missouri-Kansas City at Truman Medical Center, Kansas City, MO
}

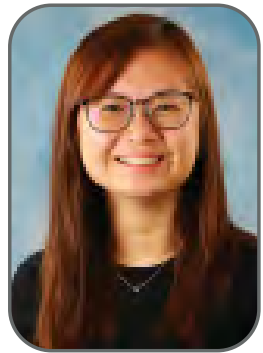

Yinglin Gao, DO

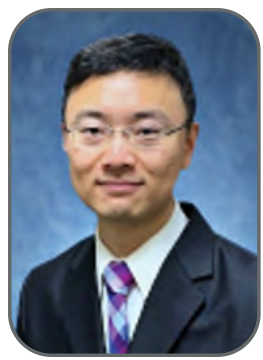

Hai Song Kim, DO

\section{KEYWORDS:}

Duodeno-Duodenal, Intussusception, Malrotation, Tubular Adenoma

\section{ABSTRACT}

Adult small bowel intussusception is a very rare entity that accounts for $5 \%$ of all cases of intussusception and $1 \%-5 \%$ of intestinal obstructions. It is more common in children but can occur in adults. It is an important etiology to consider when a patient presents with recurrent abdominal pain. The diagnosis can be challenging as symptoms are nonspecific and include abdominal pain, nausea and vomiting. This paper presents a rare case of duodenal intussusception, followed by a review of the literature discussing the diagnosis and treatment of adult intussusception.

\section{INTRODUCTION}

Intussusception is the telescoping of a part of the intestine into a more distal segment and is most commonly $h$ seen in children. Adult intussusception is very rare and represents only $5 \%$ of all intussusception cases. ${ }^{1}$ Duodenal intussusception is even rarer because of its fixed, retroperitoneal position. ${ }^{2}$ There were only 48 reported cases of duodenal intussusception since Sunderlin first described it in $1830 .^{3}$ Out of these 48 reported cases, the majority are gastroduodenal or distal duodenojejunal intussusceptions. ${ }^{3}$ We are reporting a rare case of a 57-year-old African American female who presents with duodeno-duodenal intussusception.

\section{CASE DESCRIPTION}

Our patient is a 57-year-old African American female with a past medical history significant for rheumatoid arthritis and fibromyalgia who presents to the emergency room (ER) complaining of a two-day history of intermittent LUQ and epigastric pain. The patient describes the pain as acute onset crampy sensation that "comes in waves," rated 10 out of 10 in pain scale severity, with nonspecific regional radiation; aggravated with food and associated with nausea and two episodes of non-bloody vomiting. She admits to poor oral intake but denies any hematochezia, hematemesis, melena or similar past episodes. The last bowel movement was reported a day before arrival and the patient denied any flatus. Vital signs upon admission were within normal limits. On physical examination, 
the abdomen was soft, non-distended with epigastric tenderness. Blood work on admission was unremarkable except for a white blood cell count of $11.6 \times 10^{3} / \mu \mathrm{L}$ and lactate level of $1.6 \mathrm{mmol} / \mathrm{L}$. CT Abdomen with oral contrast showed malrotation of the bowel and telescoping of the apparent second and third portions of the duodenum, forming a "target sign" compatible with intussusception. The duodenal mass served as a lead point with a fluid-filled duodenal diverticulum measuring approximately $2.4 \mathrm{~cm}$ (Figure 1).

An esophagogastroduodenoscopy was then performed and demonstrated a D2/D3 polypoid mass (Figure 2). A biopsy was obtained and showed fragments of duodenal tubular adenoma with focal moderate nonspecific chronic inflammation. No high-grade dysplasia was seen. The patient's symptoms resolved two days after presentation with supportive care. The patient then underwent endoscopic submucosal dissection of the lesion. However, the patient was lost to follow up at outpatient appointments.

\section{DISCUSSION}

Intussusception is the telescoping of a portion of the bowel into the distal portion of the adjacent bowel. Contrary to child intussusception, which is more common and usually idiopathic, adult intussusception is rare and often associated with etiologies such as a polyp, malignant neoplasm, colonic diverticulum, stricture, benign neoplasm or adenocarcinoma in the bowel wall. Of those lesions mentioned above, malignant neoplasm accounts for $66 \%$ of colonic intussusceptions and $30 \%$ of the small intestine cases. Adenocarcinoma is the most common malignant lesion in the colon, whereas metastasis is the most common malignant lead point in the small intestine ${ }^{4}$ In our case, the lead point was found to be a tubular adenoma.

Duodenal intussusception is extremely rare due to its fixed, retroperitoneal position. The exact mechanism is unclear; however, it is hypothesized that these lesions act as the lead point and alter normal peristaltic activity, which can induce invagination. ${ }^{5,6}$ Telescoping of the lead point through the distal bowel lumen could happen when the bowel's peristaltic activity produces an area of constriction above

\section{FIGURE 1.}

CT of the abdomen showing telescoping of the apparent $2 n d$ and $3 r d$ portions of the duodenum compatible with intussusception (cross-sectional plane)

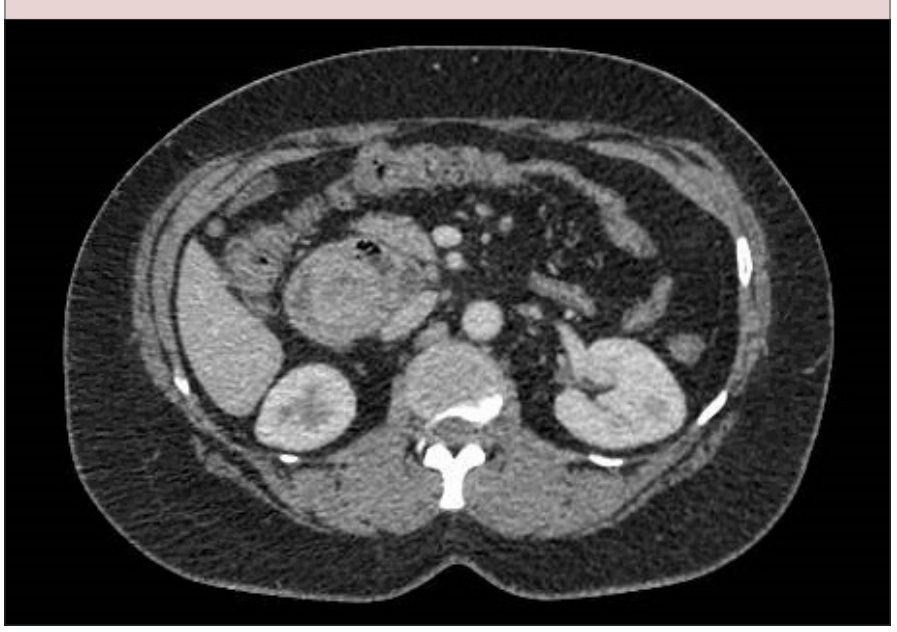

\section{FIGURE 2.}

Endoscopic image of a polypoid lesion at D2/D3 region.

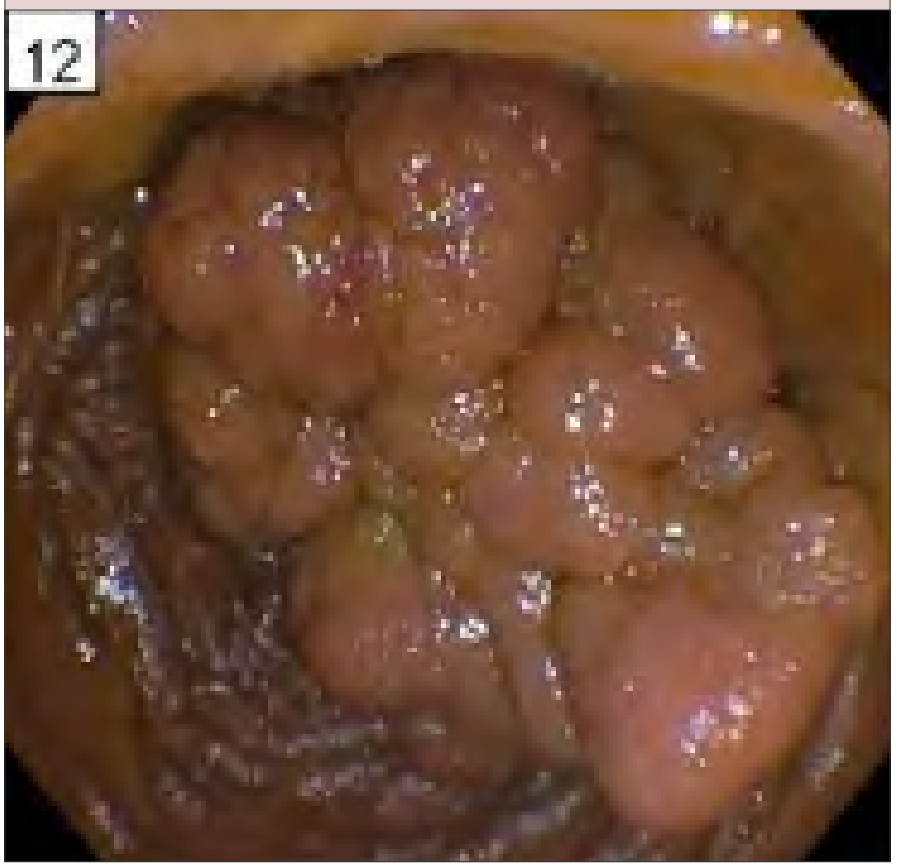


the stimulus and relaxation below. ${ }^{4}$ In addition, malrotation of the bowel can further increase the risk of intussusception, as seen in our case. ${ }^{7}$

Diagnosing adult intussusception clinically can be quite challenging because of the nonspecific symptoms such as periodic, intermittent abdominal pain, vomiting and rectal bleeding. These nonspecific symptoms are present in up to $71 \%-90 \%$ of patients. ${ }^{8}$ Abdominal CT is the most useful imaging modality and was found to have a sensitivity of up to $100 \%$ and specificity of up to $87 \%$ in adults. 9,10 The classic findings on abdominal CT are a "target sign" on the transverse axis and a sausage-shaped mass on the longitudinal axis. ${ }^{8}$ Plain abdominal films are neither sensitive nor specific. In addition, ultrasound can be useful when there is a palpable abdominal mass but may be limited by obesity and bowel gas, which may obscure typical findings. ${ }^{6}$ Other options such as colonoscopy or small bowel enteroscopy may be useful in patients with bowel obstruction. It not only confirms the intussusception but also helps with localization and tissue sampling. An esophagogastroduodenoscopy was performed and a polypoid mass was located in the D2/D3 region. The biopsy revealed tubular adenoma with moderate chronic inflammation. Our patient underwent endoscopic dissection of the lesion and was discharged home with a scheduled outpatient appointment for follow-up.

As for treatment, a surgical approach by laparotomy or laparoscopy is generally recommended and the location and pathological characteristics of the lesion should always be considered; submucosal dissection is another option as in our case. In addition, preoperative reduction by barium or air is not recommended due to the risk of perforation, seeding of microorganisms or tumor cells and increased surgical complications of manipulated friable and edematous bowel; however, it can be considered in cases where a diagnosis of the benign lesion has previously been established and the bowel involved is viable.

\section{CONCLUSION}

Adult intussusception is rare and the diagnosis is challenging due to its nonspecific symptoms. Early recognition is critical to the institution of appropriate therapy and prevention of complications as delays in treatment can lead to bowel obstruction, ischemia or intraluminal bleeding. ${ }^{10}$ Therefore, intussusception should be considered in the differential diagnosis of abdominal pain, vomiting and rectal bleeding.

\section{AUTHOR DISCLOSURES:}

No relevant financial affiliations or conflicts of interest.

\section{REFERENCES:}

1. Azar T, Berger DL. Adult intussusception. Ann Surg. 1997;226:134-138.

2. O'Connor PA, McGrath FP, Lane BE. Duodenal intussusception secondary to an internal duodenal duplication. Clin Radiol 1999;54:69-70.

3. Pradhan D, Kaur N, Nagi B. Duodenoduodenal intussusception: Report of three challenging cases with literature review. J Can Res Ther 2015;11:1031.

4. Yakan S, Calıskan C, Makay O, Deneclı AG, Korkut MA. Intussusception in adults: Clinical characteristics, diagnosis and operative strategies. World J Gastroenterol 2009; 15(16): 1985-1989.

5. Marinis A, Yiallourou A, Samanides L, et al. Intussusception of the bowel in adults: a review. World J Gastroenterol. 2009;15(4):407-411. doi:10.3748/wjg.15.407

6. Lu T, Chng YM. Adult intussusception. Perm J. 2015;19(1):79-81. doi:10.7812/TPP/14-125

7. Gardner-Thorpe J, Hardwick RH, Carroll NR, Gibbs P, Jamieson NV, Praseedom RK. Adult duodenal intussusception associated with congenital malrotation. World J Gastroenterol. 2007;13(28):38923894. doi:10.3748/wjg.v13.i28.3892

8. Yalamarthi S, Smith RC. Adult intussusception: case reports and review of literature. Postgraduate Medical Journal 2005;81:174-177.

9. Hamid Ghaderi, Ali Jafarian, Ali Aminian, Seyedeh Adeleh Mirjafari Daryasari, Clinical presentation, diagnosis and treatment of adult intussusception, a 20-years survey. International Journal of Surgery, Volume 8, Issue 4, 2010, Pages 318-210.

10. Mahmoud A. Amr, Stephanie F. Polites, Mohammad Alzghari, Edwin O. Onkendi, Travis E. Grotz, Martin D. Zielinski, Am J Surg. Author manuscript; available in PMC 2017 Jan 19. 